\title{
Trennung oder Scheidung: ehegüterrechtliche Gestaltungsmöglichkeiten
}

\author{
H. Zehntnera, J.-P. Ceccon ${ }^{b}$
}

\section{Einleitung}

Es lässt sich statistisch erhärten, dass die Scheidungsrate von Ärzten überdurchschnittlich hoch ist. In den USA liegt diese Rate mittlerweile zwischen 50 und $60 \%$. Gemäss einer neueren Studie von Guy Bodenmann, Professor für Klinische Beziehungspsychologie an der Universität Freiburg i.Ü. und Direktor des dortigen Instituts für Familienforschung und -beratung, geben in der Schweiz 47\% der befragten Ärzte an, eine nicht zufriedenstellende Partnerschaft zu führen.

Ohne an dieser Stelle auf die Gründe für die anscheinend übermässig problembelasteten Partnerschaften von Medizinern eingehen $\mathrm{zu}$ können, ist festzuhalten, dass neben den menschlichen auch die wirtschaftlichen Folgen dieser Tendenz nicht zu vernachlässigen sind. Es entspricht einer Binsenweisheit, dass eine Scheidung - oder auch eine Ehetrennung - in finanzieller Hinsicht grosse Herausforderungen an die Betroffenen stellt. Nachfolgend soll dargestellt werden, inwiefern durch vertragliche Regelungen zwischen den Ehegatten mögliche Streitpunkte vermieden oder mindestens reduziert werden können.

\section{Scheidung und Ehetrennung}

Das Zivilgesetzbuch kennt neben der Ehescheidung die Möglichkeit einer gerichtlichen Trennung, wobei die Bestimmungen über das Scheidungsverfahren auf die Ehetrennung sinngemäss anwendbar sind. Mit der Trennung tritt von Gesetzes wegen Gütertrennung ein, womit der bisherige Güterstand in aller Regel auseinandergesetzt werden muss (vorbehalten bleiben die Fälle, in welchen bereits Gütertrennung besteht). Da die hier interessierenden Fragen für beide Fälle der Beendigung der Ehe gleich zu beantworten sind, wird im folgenden nur auf den weitaus häufigeren Fall der Ehescheidung eingegangen.

\section{Wirtschaftliche Folgen}

\section{Nacheheliche Unterhaltsbeiträge}

Im Rahmen des Scheidungsurteils werden allfällige nacheheliche Unterhaltsbeiträge an den anderen Ehegatten und die Kinder festgelegt. Massgebend für die Zusprechung solcher Beiträge ist in erster Linie, ob es dem anspruchsberechtigten Ehegatten zuzumuten ist, für den gebührenden Unterhalt unter Einschluss einer angemessenen Altersvorsorge selber aufzukommen. Der Richter, welcher über Höhe und Dauer von Unterhaltsleistungen befinden muss, wird hierbei zum Beispiel berücksichtigen, welche Aufgabenverteilung während der Ehe gelebt wurde, wie lange die Ehe gedauert hat, an welchen Lebensstandard sich die Ehegatten gewöhnt haben, welches Einkommen und Vermögen vorhanden ist usw.

Festzuhalten ist, dass der Richter die Beiträge, welche in aller Regel in Form einer Rente zu erbringen sind, gestützt auf objektive Kriterien festsetzt. Das unsägliche Verschuldensprinzip, welches eine «strafende Note» in die Bemessung der Beiträge einbrachte und regelrecht als «Brandbeschleuniger» nicht weniger Scheidungsverfahren diente, wurde mit den revidierten Bestimmungen, welche seit dem Jahr 2000 in Kraft sind, eliminiert.

Die Parteien können im Rahmen einer sogenannten Nebenfolgenkonvention die Unterhaltsbeiträge regeln. Diese muss jedoch dem Gericht eingereicht und im Rahmen des Scheidungsurteils bewilligt werden. Die Festsetzung ist demnach der Disposition der Parteien entzogen, sie können nur dem Gericht einen Vorschlag unterbreiten. Es wäre demnach verfehlt, in diesem Kontext von vertraglichen Gestaltungsmöglichkeiten zu sprechen.

\section{Ehegüterrecht}

Mit der Scheidung findet zwingend eine güterrechtliche Auseinandersetzung statt. Der bestehende Güterstand wird damit aufgelöst, und die Vermögenswerte werden nach gesetzlicher Vorschrift oder aber gestützt auf die Abmachungen 
in einem allfälligen Ehevertrag verteilt. Das schweizerische Recht kennt drei mögliche Güterstände: Neben dem ordentlichen Güterstand der Errungenschaftsbeteiligung können die Ehegatten ehevertraglich den Güterstand der Gütergemeinschaft oder der Gütertrennung wählen. Der Vollständigkeit halber sei erwähnt, dass die Gütertrennung auch kraft Gesetzes eintreten oder aber per Urteil herbeigeführt werden kann.

Im folgenden soll auf den ordentlichen Güterstand der Errungenschaftsbeteiligung, welcher einerseits naturgemäss der am weitesten verbreitete ist und anderseits die interessantesten Gestaltungsmöglichkeiten bietet, näher eingegangen werden.

In diesem Güterstand haben beide Ehegatten je ihr Eigengut und ihre Errungenschaft (es gibt also vier Gütermassen). Dem Eigengut gehören vereinfacht die Vermögenswerte an, welche in die Ehe eingebracht werden und welche während der Dauer der Ehe unentgeltlich erworben werden (insbesondere Schenkungen und Erbschaften). Der Errungenschaft gehören demgegenüber die Vermögenswerte an, welche während der Dauer der Ehe entgeltlich erworben werden (insbesondere Arbeitserwerb). Ohne abweichende ehevertragliche Regelung erhält jeder Ehegatte bei der Auflösung des Güterstandes die Hälfte der Errungenschaft des Ehepartners und behält sein Eigengut.

Der gesetzlich gewährte Gestaltungsspielraum muss regelmässig in ehevertraglicher Form wahrgenommen werden, was eine öffentliche Beurkundung bedingt. Um den Rahmen dieses Beitrags nicht unnötig zu strapazieren, sei an dieser Stelle nur auf die folgenden Möglichkeiten hingewiesen:

- Erträge des Eigenguts können dem Eigengut gewidmet werden (ohne Regelung fallen sie in die Errungenschaft).

- Die hälftige Teilung der Errungenschaft kann geändert werden. Es kann also einem Ehegatten die ganze Errungenschaft zugewiesen werden. In aller Regel gilt dies nur für den Fall der Auflösung des Güterstandes infolge Todes.
Soll es auch für die Scheidung gelten, muss dies explizit im Ehevertrag vorgesehen sein.

- Es kann ehevertraglich vereinbart werden, dass Vermögenswerte der Errungenschaft, welche für die Ausübung eines Berufs bestimmt sind, dem Eigengut eines Ehepartners angehören.

Insbesondere der letzte Punkt mag erhellen, dass mittels vertraglicher Regelung zum Beispiel eine Arztpraxis aus der güterrechtlichen Auseinandersetzung «ausgeklammert» werden kann. Dies setzt jedoch voraus, dass sich die Ehegatten rechtzeitig mit diesen doch eher unangenehmen Fragen auseinandersetzen und zu einer vertraglichen Regelung finden.

Die Zuweisung der ganzen Errungenschaft an den anderen Ehegatten ist eine häufig verwendete Möglichkeit, den überlebenden Ehegatten gegenüber den Nachkommen zu begünstigen. Dies kann zum Beispiel sicherstellen, dass die eheliche Liegenschaft nicht veräussert werden muss, um die Ansprüche der Kinder befriedigen zu können. Solche Begünstigungen werden in aller Regel in der Form eines kombinierten Eheund Erbvertrages realisiert, was weitere Gestaltungsmöglichkeiten eröffnet. $\mathrm{Zu}$ nennen sind etwa eine zusätzliche erbrechtliche Begünstigung des überlebenden Ehegatten unter Wahrung der Pflichtteilsrechte der Nachkommen oder aber eine lebenslängliche Nutzniessung des überlebenden Ehegatten am gesamten den Nachkommen zufallenden Nachlass. Vor der Realisierung allfälliger Gestaltungsmöglichkeiten ist eine eingehende Analyse der angestrebten Lösung zu empfehlen; von Standardlösungen ist gerade in anspruchsvolleren Kontexten abzuraten.

Im Sinne eines Fazits lässt sich festhalten, dass eine Überprüfung der möglicherweise bereits getroffenen Dispositionen im Bereich Eheund Erbrecht und allenfalls die Ausarbeitung einer optimierten Regelung in vielen Fällen Sinn macht. 